Available online at http://iddtonline.info
Journal of Drug Delivery and Therapeutics
Open access to Pharmaceutical and Medical research
(C) 2014, publisher and licensee JDDT, This is an Open Access article which permits unrestricted noncommercial use, provided the original
work is properly cited

REVIEW ARTICLE

\title{
SKELETAL DRUG DELIVERY SYSTEM: A REVIEW
}

\author{
Kar Mousumi*, Singh Sourabh, Jain Dinesh Kumar \\ College of Pharmacy, IPS Academy, Indore, M.P., India, 452012 \\ *Corresponding Author's Email id: karmousumi@hotmail.com
}

\begin{abstract}
:
Drug delivery refers to approaches, formulations, technologies, and systems for transporting a pharmaceutical compound in the body as needed to safely achieve its desired therapeutic effect. Skeletal drug delivery systems (SDDS) are used to deliver the drug directly to skeletal tissue through various conventional and novel approaches, thereby improving the therapeutic effectiveness of drugs in bone diseases. It may involve scientific site-targeting within the body, or it might involve facilitating systemic pharmacokinetics; in any case, it is typically concerned with both quantity and duration of drug presence.

Ranging from use of poultice and now with the advent of self setting cements, nanoparticles, liposomes etc for targeting the drug to the poorly perfused body organs, the development of delivery systems to bones/ skeletal system have come a long way. The present work reviews the delivery systems that are prevalent and is dedicated towards a rationale selection of the drugs and dosage forms for skeletal diseases.

Key words: Skeletal drug delivery systems, conventional, novel, drugs used.
\end{abstract}

\section{INTRODUCTION}

Drug delivery is often approached via a drug's chemical formulation, but it may also involve medical devices or drug-device combination products. Drug delivery is a concept heavily integrated with dosage form and route of administration. These technologies modify drug release profile, absorption, distribution and elimination for the benefit of improving product efficacy and safety, as well as patient convenience and compliance. Drug release is from: diffusion, degradation, swelling, and affinity-based mechanisms.

Current efforts in the area of drug delivery include the development of targeted delivery in which the drug is only active in the target area of the body (for example, in cancerous tissues) and sustained release formulations in which the drug is released over a period of time in a controlled manner from a formulation. In order to achieve efficient targeted delivery, the designed system must avoid the host's defense mechanisms and circulate to its intended site of action. Types of sustained release formulations include liposomes, drug loaded biodegradable microspheres and drug polymer conjugates.

\section{Skeletal Drug Delivery System:}

Selective drug delivery to any organ becomes very important in certain diseases and clinical manifestations, especially when the drug affects other exposed tissues adversely. The importance of selective drug action is still further increased when the affected part is poorly perfused. Although a network of blood vessels is present throughout skeletal tissue (or bone), it is not sufficient for immediate delivery of drugs to the desired site of action in the tissue and in sufficient amounts over (C) 2011-14, JDDT. All Rights Reserved appropriate time periods. Hence, selective drug delivery to the skeletal system has remained a great challenge to pharmaceutical scientists over the years. However, in the recent past, attention has been focused on the importance of skeletal drug delivery and the first Skeletal Drug Delivery System (SDDS) was introduced by Bucholz and Engelbrecht in 1970 for the delivery of drugs to skeletal tissues at a high concentration to achieve desirable therapeutic effects. ${ }^{1}$

\section{Skeletal Drug Delivery and Tissue Engineering:}

Protein and peptide delivery and nanotechnology have been applied in skeletal drug delivery (targeting to bones and cartilages). Composite tablets, implants, and injectable hydrogels composed of biodegradable polymers and ceramics are developed as locally deliver systems of antibiotics, chemotherapeutic agents, steroids, non-steroid anti-inflammatory drugs, etc. Their physicochemical characteristics, product stability, bioactivities, are characterized. Release profiles, antimicrobial activities and biocompatibility of the composites are evaluated in vitro. Targeted delivery systems of growth factors and protein drugs to bones and cartilages may be suitable in to create new tissues from the cultured cells. It has been considered as a potential alternative to organ or tissue transplantation. Biodegradable scaffolds and suitable growth factor delivery systems play important roles in transforming the cultured cells to new tissues. Modification of material surface with cell specificity can be grafted to scaffolds to prepare cell specific scaffolds. Cultures of osteoblast-like cells and chondrocytes conditioned with controlled release delivery systems and suitable therapeutic forms 
of growth factors can promote cell proliferation and differentiation in bone and cartilage tissue engineering.

\section{Anatomy of Skeletal System:}

Skeletal system is all of the bones in the body and the tissues such as tendons, ligaments and cartilage that connect them. The skeletal system includes all of the bones and joints in the body. Each bone is a complex living organ that is made up of many cells, protein fibres, and minerals. The skeleton acts as a scaffold by providing support and protection for the soft tissues that make up the rest of the body. The skeletal system also provides attachment points for muscles to allow movements at the joints. New blood cells are produced by the red bone marrow present inside the bones. Bones act as the body's warehouse for calcium, iron, and energy in the form of fat. Finally, the skeleton grows throughout childhood and provides a framework for the rest of the body to grow along with it.

\section{Anatomy:}

The skeletal system in an adult body is made up of 206 individual bones. These bones are arranged into two major divisions: the axial skeleton and the appendicular skeleton. The axial skeleton runs along the body's midline axis and is made up of 80 bones in the following regions: Skull, auditory ossicles, ribs, sternum and vertebral column. The appendicular skeleton is made up of 126 bones in the regions: upper and lower limbs, pelvic and pectoral girdle.

\section{Microscopic structure of Bone:}

The skeleton makes up about $30-40 \%$ of an adult's body mass. The skeleton's mass is made up of nonliving bone matrix and many tiny bone cells. Roughly half of the bone matrix's mass is water, while the other half is collagen protein and solid crystals of calcium carbonate and calcium phosphate.

Living bone cells are found on the edges of bones and in small cavities inside of the bone matrix. Although these cells make up very little of the total bone mass, they have several very important roles in the functions of the skeletal system ${ }^{2}$. The bone cells allow bones to:

- Grow and develop

- Be repaired following an injury or daily wear

- Be broken down to release their stored minerals

Old bones are dead, dry and brittle. But in the body, bones are very much alive. They have their own nerves and blood vessels, and they do various jobs, such as storing body minerals like calcium. Bones are made of a mix of hard stuff that gives them strength and tons of living cells which help them grow and repair themselves.

\section{Skeletal System Physiology:}

$>$ Support and Protection: The skeletal system's primary function is to form a solid framework that supports and protects the body's organs and anchors the skeletal muscles. The bones of the axial skeleton act as a hard shell to protect the internal organssuch as the brain and the heart-from damage caused by external forces. The bones of the appendicular skeleton provide support and flexibility at the joints and anchor the muscles that move the limbs.

$>$ Movement: The bones of the skeletal system act as attachment points for the skeletal muscles of the body. Almost every skeletal muscle works by pulling two or more bones either closer together or further apart.

$>$ Hematopoiesis: Red bone marrow produces red and white blood cells in a process known as hematopoiesis. Red bone marrow is found in the hollow space inside of bones known as the medullary cavity. ${ }^{4}$

> Storage: The skeletal system stores many different types of essential substances to facilitate growth and repair of the body. The skeletal system's cell matrix acts as our calcium bank by storing and releasing calcium ions into the blood as needed. Proper levels of calcium ions in the blood are essential to the proper function of the nervous and muscular systems. Bone cells also release osteocalcin, a hormone that helps regulate blood sugar and fat deposition. The yellow bone marrow inside of our hollow long bones is used to store energy in the form of lipids. Finally, red bone marrow stores some iron in the form of the molecule ferritin and uses this iron to form hemoglobin in red blood cells.

> Growth and Development: The skeleton begins to form early in fetal development as a flexible skeleton made of hyaline cartilage and dense irregular fibrous connective tissue. These tissues act as a soft, growing framework and placeholder for the bony skeleton that will replace them. As development progresses, blood vessels begin to grow into the soft fetal skeleton, bringing stem cells and nutrients for bone growth. Osseous tissue slowly replaces the cartilage and fibrous tissue in a process called calcification. The calcified areas spread out from their blood vessels replacing the old tissues until they reach the border of another bony area.

\section{Disorders of skeletal system:}

\section{Osteoporosis:}

Osteoporosis is a disease of the bones, due to loss of bone or very little bone or both. Osteoporosis means "porous bone". A healthy bone observed under a microscope, parts of bone look like a honeycomb. If bone of a person suffering from osteoporosis is examined under a microscope, the holes and spaces in the honeycomb are much bigger than they are in healthy bone. This means bones have lost density or mass and that the structure of bone tissue has become abnormal. If bones become less dense, they also become weaker and more likely to break. Nearly $60 \%$ of adults age 50 and older are at risk of breaking a bone and should be concerned about bone health. Studies also suggest that approximately one in two women and up to one in four men age 50 and older will break a bone due to osteoporosis. 
Osteoporosis is often called the "silent disease," because people could have it now or be at-risk without even realizing it. Osteoporosis affects men and women of all races. But white and Asian women especially those who are past menopause are at highest risk. Medications, healthy diet and weight-bearing exercise can help prevent bone loss or strengthen already weak bones.

\section{Bursitis:}

Bursitis is the inflammation of one or more bursae (small sacs) of synovial fluid in the body. Healthy bursae create a smooth, almost frictionless functional gliding surface making normal movement painless. When bursitis occurs, however, movement relying upon the inflamed bursa becomes difficult and painful. Moreover, movement of tendons and muscles over the inflamed bursa aggravates its inflammation, perpetuating the problem. Muscle can also be stiffened. Bursitis is commonly caused by repetitive movement and excessive pressure. Shoulders, elbows and knees are the most commonly affected. Traumatic injury is another cause of bursitis. The inflammation irritates because the bursa no longer fits in the original small area between the bone and the functionary muscle or tendon.

\section{Osteoarthritis:}

Osteoarthritis (OA) also known as degenerative arthritis or degenerative joint disease or osteoarthrosis, is a group of mechanical abnormalities involving degradation of joints, including articular cartilage and subchondral bone. Symptoms may include joint pain, tenderness, stiffness, locking, and sometimes an effusion. A variety of causes-hereditary, developmental, metabolic, and mechanical deficits - may initiate processes leading to loss of cartilage. When bone surfaces become less well protected by cartilage, bone may be exposed and damaged. As a result of decreased movement secondary to pain, regional muscles may atrophy, and ligaments may become more lax.

\section{Osteomyelitis:}

Osteomyelitis (sometimes abbreviated to OM, and derived from Greek words osteon, meaning bone, myelomeaning marrow, and -itis meaning inflammation) is infection and inflammation of the bone or bone marrow. It can be usefully sub-classified on the basis of the causative organism (pyogenic bacteria or mycobacteria), the route, duration and anatomic location of the infection. $^{3}$

\section{Paget's Disease:}

Paget's disease of bone is a chronic disorder that can result in enlarged and misshapen bones. Paget's is caused by the excessive breakdown and formation of bone, followed by disorganized bone remodeling. This causes affected bone to weaken, resulting in pain, misshapen bones, fractures, and arthritis in the joints near the affected bones. Paget's disease typically is localized, affecting just one or a few bones, as opposed to osteoporosis, for example, which usually affects all the bones in the body. ${ }^{5}$

\section{DRUGS INCORPORATED IN SKELETAL DRUG DELIVERY DEVICES/SYSTEM:}

\section{Norfloxacin:}

Norfloxacin is a synthetic chemotherapeutic antibacterial agent. In form of ophthalmic solutions it is known as Chibroxin. Norfloxacin is a first generation synthetic fluoroquinolone (quinolone) developed by Kyorin Seiyaku K.K. (Kyorin). ${ }^{5}$ Norfloxacin interacts with a number of other drugs, as well as a number of herbal and natural supplements. Such interactions increase the risk of anticoagulation and the formation of non-absorbable complexes, as well as increasing the risk of toxicity.

Norfloxacin is a broad-spectrum antibiotic that is active against both Gram-positive and Gram-negative bacteria. It functions by inhibiting DNA Gyrase, a type II topoisomerase, and topoisomerase IV,enzymes necessary to separate bacterial DNA, thereby inhibiting cell division. $^{6,7}$

\section{Cefalexin:}

Cefalexin (INN) or more commonly cephalexin is a firstgeneration cephalosporin antibiotic introduced in 1967 by Eli Lilly and Company. It is an orally administered agent with a similar antimicrobial spectrum to the intravenous agents cefalotin and cefazolin. It was first marketed as Keflex (Lilly), and is marketed under several other trade names. Cefalexin is used to treat a number of infections including: otitis media, streptococcal pharyngitis, bone and joint infections, pneumonia, cellulitis, and urinary tract infections.

\section{Samarium-153:}

Samarium-153 is an isotope of samarium. It emits beta particles and gamma rays. The therapeutic component is largely due to the beta particles, but the gamma rays make it easier to locate the distribution. It is used in samarium $\left({ }^{153} \mathrm{Sm}\right)$ lexidronam. It is treated by the body in a similar manner to calcium, and it localizes selectively to bone. It is used in palliation of bone cancer.

\section{Samarium $\left({ }^{153} \mathrm{Sm}\right)$ Lexidronam:}

Samarium $\left({ }^{153} \mathrm{Sm}\right)$ lexidronam (chemical name Samarium-153-ethylenediaminetetramethylene phosphonate, abbreviated Samarium-153 EDTMP, trade name Quadramet) is a complex of a radioisotope of the lanthanideelementsamarium with the chelator EDTMP. It is used to treat pain when cancer has spread to the bone. $8,9,10,11$ It is injected into a vein and distributed throughout the body. It is preferentially absorbed in areas where cancer has invaded the bone. There the radioisotope emits beta particles (electrons) which kill the nearby cancer cells. Pain begins to improve in the first week for most people and the effects can last several months. It is commonly used in lung cancer, prostate cancer, breast cancer, and osteosarcoma. ${ }^{12,13}$ 
Table 1: Drugs used for treatment of disorders of skeletal system

\begin{tabular}{|l|l|l|}
\hline S. No. & Drug Name & Uses (Disease) \\
\hline 1. & Norfloxacin & Spontaneous tendon ruptures \\
\hline 2. & Cefalexin & $\begin{array}{l}\text { Bacterial endocarditis (pneumonia, urinary tract inf., bone\& joint } \\
\text { inf., otitis media). }\end{array}$ \\
\hline 3. & Samarium-153 & Palliation (bone cancer). \\
\hline 4. & Samarium(153SM) lexidronam & Lung cancer, prostate cancer, breast cancer (Osteosarcoma). \\
\hline
\end{tabular}

\section{Biocomposites: Bone Cement Composites, Hydroxyapatite and Biomimetic Hybrid Composites:}

A biocomposite is a material formed by a matrix (resin) and a reinforcement of natural fibers (usually derived from plants or cellulose). They often mimic the structures of the living materials involved in the process in addition to the strengthening properties of the matrix that was used but still providing biocompatibility, e.g. in creating scaffolds in bone tissue engineering ${ }^{14}$. Biocomposites are characterized by the fact that:

$>$ the petrochemical resin is replaced by a vegetable or animal resin, and/or

$>$ the bolsters (fiberglass, carbon fiber or talc) are replaced by natural fiber (wood fibers, hemp, flax, sisal, jute)

Bone Cement Composites: During the last 5 years bone cement materials have grown in popularity and are very promising osteoconductive substitutes for bone graft. They are prepared like acrylic cements and contain a range of powders such as monocalcium phosphate, tricalcium phosphate and calcium carbonate, which is mixed in a solution of sodium phosphate. These cements are produced without polymerization and the reaction is nearly non-exothermic. The final compounds are reported to have a strength of $10-100 \mathrm{MPa}$ in compression while 1-10 MPa in tension, although very weak under shear forces. These composites are currently used in orthopedics in the management of fractures. Injection of calcium phosphate cement has been shown to be feasible and it does improve their compressive strength.

Hydroxyapatite Composite Materials: Hydroxyapatite cement is a calcium phosphate-based material that when mixed with water forms a dense pastes that sets within 15 minutes and isothermically converts in vivo to a micro porous hydroxyapatite implant. Preparation of hydroxyapatite/ceramic composites through the addition of various ceramic reinforcements has been attempted, metal fibers, $\mathrm{Si}_{3} \mathrm{~N}_{4}$ or hydroxyapatite whiskers, $\mathrm{Al}_{2} \mathrm{O}_{3}$ platelets and $\mathrm{ZrO}_{2}$ particles. Hydroxyapatite/metal and hydroxyapatite/polymer composites are two typical classes of materials, which have been examined for improving the toughness characteristics of synthetic hydroxyapatite. In both cases, a toughness improvement can be found, due to a crack-face bridging mechanism operated upon plastic stretching of metallic or polymeric ligaments. Zhang et al. proposed a toughened composite consisting of calcium hydroxyapatite dispersed with silver particles. This material was obtained by a conventional sintering method. It was reported that the toughness of these composites increased up to $2.4 \mathrm{MPa}$ $\mathrm{m}^{1 / 2}$ upon loading the mixture, with (30 vol. \%) silver ${ }^{15}$. (c) 2011-14, JDDT. All Rights Reserved
Biomimetic Hybrid Composites: The conventional way to synthesize an inorganic material-based composite is to subject a mixture of the constituent phases to heat treatment. Traditional sintering route is not directly applicable to produce ceramic/polymer composites because no polymer will stand at the densification temperature of any ceramic material. Hydroxyapatite/polyethylene composites have been obtained by loading the polymeric matrix with the inorganic filler. In recent years, several research groups have demonstrated the feasibility of in vitro techniques for the synthesis of biomimetic material structures. A common characteristic of natural biomaterials such as bone, nacre, sea urchin tooth and other tough hybrid materials in nature is the strong microscopic interaction between the inorganic and the organic phases. This characteristic allows the organic phase to act as a plastic energy-dissipating network, forming stretching (bridging) ligaments across the faces of a propagating crack in a nano-scale level. Such complexity has led to the common perception that, to mimic natural designs, in situ synthesis techniques should be adopted. Precipitation of calcium carbonate or hydroxyapatite into a polymeric matrix, for example, has been proposed as a novel synthetic route to biomimetic composites.

\section{APPROACHES FOR SKELETAL DRUG DELIVERY SYSTEMS:}

\section{Calcium Phosphate Cements As Bone Drug Delivery Systems:}

Since calcium phosphate cements were proposed, several formulations have been developed, some of them commercialized, and they have proven to be very efficient bone substitutes in different applications. Some of their properties, such as the inject ability, or the lowtemperature setting, which allows the incorporation of different drugs, make them very attractive candidates as drug carriers. ${ }^{14,16}$

\section{Calcium phosphate:}

Calcium phosphate is the name given to a family of minerals containing calcium ions $\left(\mathrm{Ca}^{2+}\right)$ together with orthophosphates $\left(\mathrm{PO}_{4}{ }^{3-}\right)$, meta-phosphates or pyrophosphates $\left(\mathrm{P}_{2} \mathrm{O}_{7}{ }^{4-}\right)$ and occasionally hydrogen or hydroxide ions. Calcium phosphate is the principal form of calcium found in bovine milk. Seventy percent of bone consists of hydroxyapatite, a calcium phosphate mineral (known as bone mineral). Tooth enamel is composed of almost ninety percent hydroxyapatite.

$>$ Calcium phosphates:

- Monocalcium phosphate, E341(i): $\mathrm{Ca}\left(\mathrm{H}_{2} \mathrm{PO}_{4}\right)_{2}$

- Dicalcium phosphate, E341(ii): $\mathrm{CaHPO}_{4}$ 
- Tricalcium phosphate (or tricalcic phosphate), E341(iii): $\mathrm{Ca}_{3}\left(\mathrm{PO}_{4}\right)_{2}$, sometimes referred to simply as calcium phosphate or calcium orthophosphate

- Hydroxyapatite $\mathrm{Ca}_{5}\left(\mathrm{PO}_{4}\right)_{3}(\mathrm{OH})$

- Apatite $\mathrm{Ca}_{10}\left(\mathrm{PO}_{4}\right)_{6}(\mathrm{OH}, \mathrm{F}, \mathrm{Cl}, \mathrm{Br})_{2}$

- Octacalcium phosphate $\mathrm{Ca}_{8} \mathrm{H}_{2}\left(\mathrm{PO}_{4}\right)_{6} .5 \mathrm{H}_{2} \mathrm{O}$

\section{Calcium Phosphate Cements:}

In the field of the pharmacological treatment of skeletal disorders, the specific characteristics of bone tissue should be considered. Thus an ideal drug carrier for bone should be bioactive, which would ensure the ability of the materials to bond to the bone tissue, and resorbable to allow its progressive by newly formed bone. Additional advantages are provided if the material is injectable, since it would improve ease of administration, by allowing minimally invasive surgical techniques. All these properties are very well fitted by calcium phosphate cement, and therefore they should be competitive candidates to be used for this application.

\section{a. CPC as drug carrier material:}

The development of CPC was an important breakthrough in the field of bioceramices for bone regeneration, since it supplied a material which was mould-able and which had the capacity of self-setting in-vivo within the bone cavity. Their ability to set once implanted within the body, giving a highly microporous material, allows incorporation of many types of drugs and biologically active molecules, without losing activity and denaturalization. Therefore, the possibility to use CPCs not only as bone substitutes, but as a carrier for local and controlled supply of drugs is very attractive and can be useful in treatments of different skeletal diseases, which normally require long and painful therapies, as well as for accelerating the rate of bone fracture healing.

Calcium phosphate cements as carriers for antibiotics: In the field of application of CPCs for drug delivery, the antibiotics have been widely studied, due to their frequent use in the treatment of infections of the skeletal system, or as a prophylactic strategy. Antibiotics are often provided as prophylactics, either orally or intravenously. However, the very little accessibility of the site of infection very often prolongs the treatment of bone infections. A traditional method applied to control bone infections is the implantation of polymethylmethacrylate spheres (PMMA) loaded with gentamicin sulphate in the infection site.

Calcium phosphate cements as carriers for other drugs: anti-inflammatory, analgesic and anticancer: Besides their application as antibiotic carriers, CPC can be appropriate matrixes for the incorporation of other drugs with potential application in the musculoskeletal system. Ginebra et al. studied the effect of the incorporation of an amino salicylic acid derived methacrylamide in apatitic $\alpha$-TCP-based cement. Salicylic acid is an analgesic and anti-inflammatory, and moreover has calcium complexation ability. Strong effects were observed both in the rheological and mechanical properties. The inject ability was improved, but simultaneously a decrease in the reaction rate of the cement was observed.
Calcium phosphate cements as carriers for growth factors and other proteins: Growth factors are a large group of polypeptides, able to transmit signals which affect cellular activity. Among them, the superfamily of $\beta$-transforming growth factors (TGF $\beta$-SF) is especially relevant for bone regeneration. It includes the transforming growth factors $\beta 1, \beta 2, \beta 3$ (TGF- $\beta 1, \beta 2$ and $\beta 3$ ), and the bone morphogenetic proteins (BMP) among other proteins. It is known that they play a role as activating agents for the complex cascades of biological phenomena responsible for bone formation, and therefore they can accelerate bone in growth. ${ }^{15,16,17}$

\section{Essential oils:}

Essential oils also known in the literature as etheric oils are fragrant, highly volatile (quickly evaporating in contact with air) liquids that are produced naturally in specialized glands of aromatic plants, each essential oil are a unique energetic being.

Essential oils are valuable is because they have several things in common with human blood. They are accepted by the body because of these commonalities. They include: compatibility with human proteins, ability to fight infection and regenerate tissue, similarity in chemical structure with human cells, similar hormonelike compounds.

The essential oils used for skeletal systems include Helichrysum, Wintergreen, Peppermint, Idaho Balsam Fir, Clove, Peace \& Calming, PanAway.

Rheumatic disease and arthritis in childhood are important causes of both short and long term disability. Early medical diagnosis and treatment can minimize the short and long term evolution of this condition ${ }^{18,19,20}$.

\section{Poultice:}

This ancient, traditional heat treatment soothes and alleviates muscle tension using a combination of compresses and massage, with specific herbs chosen to purify and detoxify the body. By opening the pores of the skin, the warm medicinal herbs naturally reduce swelling and inflammation of muscles, ligaments and joints.

However, Bone/Skin Poultice is more than a "fill-in" since this supplement provides all the medicinal merits of comfrey Bone/Skin Poultice combines yarrow flowers, mullein leaf, plantain herb and Chinese rehmannia root to give additional benefits not found in comfrey alone.

\section{Herbs Used In Poultice:}

> Plantain: This famous herb helps pull tissues together for healing (astringent), is antiseptic and diuretic, in addition to cooling, soothing and healing. Plantain is excellent for repairing both acute and chronic wounds and can be used internally and externally.

> Chinese rehmannia root: In Chinese herbalism this root has been used to treat specific conditions such as-headaches, migraines, arthritis, rheumatism, flu, inflammatory skin conditions and anemia.

$>$ Mullein leaf: An aid for pain, bleeding and infection, mullein leaf is also an anti-spasmodic (relieves 
cramps). This herb is famous for lung problems and is a wonderful healer, even for swollen tissues including the joints.

> Yarrow flowers: Yarrow contains a blue volatile oil that is a powerful anti - inflammatory, so it reduces swelling, redness and speeds healing.

a. Uses of Poultice:

$>$ Poultice of clay with lemon juice: The poultice of clay mixed with lemon juice more or less diluted with water as the case has a special power in all the "itis", i.e. inflammation with evident pain, including arthritis and the like.

$>$ Poultice of clay with purple grape juice: The black grape is always preferable to white. The large amount of tannin contained in the creditor makes a special place in trofoterapia and the same here, geoterapia or cure for the land. If we use placed on the heart area is an excellent tonic, like if we apply it on the spine or joints will be an active regenerating skeletal system, i.e. the bones.

\section{Plaster:}

There are several different kinds of plaster casting material available. The choice depends on the particular injury. In a Stable fracture the cast is often only needed to protect the bone from further injury and limit movement a little at the fracture site. (A little movement stimulates healing; too much movement slows it down). Hence the molding and fit is not as critical. It is also possible to make a removable cast or use pre made splints. In an unstable fracture treated just with a cast, here the plaster cast is critical in holding the bone ends in a good position and as such the fit and molding is very important $22,23,24$.

Following an acute fracture swelling can be a problem and as such a:

a. Backslab: "Backslab" is used; this does not have to go on the back of the limb. This is

made up of an under layer of orthopaedic wool, with a slab of usually old fashioned white plaster of paris. It does not encircle the limb, usually covering half or $3 / 4 \mathrm{rs}$ of the circumference of the limb. A bandage is applied to keep it well applied to the limb. The "cut away" completed by the bandage allows for swelling. "Backslabs" are usually temporary, used for a few days. They usually do not last longer than 2 weeks.

b. Plaster of Paris (Gypsum): Plaster of paris is often used in acute, fresh fractures. The reason Plaster of paris (Gypsum) is often used in this setting, is because it is a lot easier to mould to get a snug fit without creating any pressure areas. It is heavier than and not as resilient as synthetic casts but still the preferred casting material for fractures requiring a snug fit and "molding" over the fracture to keep the bones in a good position

c. Synthetic casts: Often called fibreglass casts, this is a slight misnomer not all synthetic casts are made of fiber glass, and some are made of polyester. There are two main synthetic casting groups.
Hard casts and soft casts- Softcasts are used mostly for support or stable fractures in kids. Benefits of softcast include the ability to remove by unwrapping or cutting off the cast without the need for a plaster saw. It is also possible to manufacture a "swimming cast" using softcast.

d. Swimming cast: Plaster of paris (Gypsum) should not be placed in water it softens the cast and it will disintegrate. Synthetic casts (hard) will normally not fall apart if they get wet or splashed. The problem is the padding or underlay remains wet for a long time and the skin may macerate. If you do get your hard synthetic cast moist or wet dry it out with a hairdryer.

e. Looking after swimming cast: The problem with swimming softcasts and water is not that the cast will disintegrate but that the skin will become soggy and macerate. Flush out the limb with clean water to get rid of any dirt and wash out any pool chemicals. Dry out the limb if not in a warm climate use a hair dryer and ensure the cast dries totally. Give the skin a break does not keep it soaked in water all the time.

6. Lepa: The preparation in the form of paste meant for external application on the body is known as lepa. The crude drug in the powdered form are mixed with cow urine, water, oil or ghee and made into paste. Lepas prepared from vegetable powders are stable only for one month, while lepas containing minerals or metals long last.

Example: Sinduradi lepa, Pathyadi lepa, Dasanga lepa and Dosaghna lepa.

7. Ointments: A highly viscous or semisolid substance used on the skin as a cosmetic, emollient, or medicament. Ointment is, any greasy substance rubbed on the skin to heal injuries.

\section{NOVEL APPROACHES FOR SKELETAL DRUG DELIVERY SYSTEMS:}

\section{Self-Setting Hydroxypatite Cement:}

To solve the problem of delivering drugs to skeletal tissue at high enough local concentrations for desirable therapeutic effects, a novel approach using a self-setting hydroxyapatite cement, with cephalexin and norfloxacin as model drugs is reported. After setting, the cement was transformed into hydroxyapatite with affinity for hard bone tissue. Continuous in-vitro drug release profiles from loaded cement pellets $(0.9-4.8 \%$ by weight $)$ in phosphate buffer at $\mathrm{pH} 7.4$ and 37 degrees $\mathrm{C}$ followed the Higuchi equation. A novel approach using a selfsetting hydroxyapatite (HAP) cement as a skeletal drugdelivery system has been proposed to solve the problem of delivering drugs to skeletal tissue at sufficiently high local concentrations for desirable therapeutic effects. HAP cements loaded with antibiotics can be formed in situ and can be used as bonding materials between bone and prostheses, as well as drug-release devices. The cement also possesses sufficient mechanical strength to be a potential bone grafting material. Using cephalexin and norfloxacin as model drugs, continuous in vitro release profiles of these compounds from cement pellets 
loaded $0.9-4.8 \%$ by weight with one of the drugs is observed. This drug-release pattern correlated well with the Higuchi model. This hydroxyapatite cement drugdelivery system can be applied in the treatment of osteomyelitis and infected compound fractures. ${ }^{25,26}$

\section{Bone Specific-Drug Delivery System:}

Bone-specific drug delivery systems approache via chemical modification of bone-seeking agents. Despite several decades of progress, bone-specific delivery is still limited by the unique anatomical features of bone, which mainly consists of inorganic hydroxyapatite. A practical approach to this problem is to produce targeted drugs that have a high affinity for hydroxyapatite. Bisphosphonates are a class of synthetic compounds structurally related to pyrophosphate. Bisphosphonates rapidly localize on the bone surface after being administered either intravenously or orally, since the PC-P portion of the bisphosphonate structure has high affinity for hydroxyapatite. Therefore, bisphosphonate modification might be a promising method for targeting drugs selectively to the bone. Bisphosphonateconjugated drugs are hydrophilic and highly watersoluble due to the acidic nature of the bisphosphonate moiety at physiological $\mathrm{pH}$, and therefore they hardly permeate through the biological membrane of soft tissues. These physicochemical changes also reduce the intrinsic susceptibility of the drug to metabolism, promoting urinary or biliary excretion as unchanged drug. All these physicochemical and pharmacokinetic alterations contribute to the exceptional skeletal disposition of bisphosphonate-conjugated drugs. Although most examples of bone-specific drug delivery via bone-seeking agents still remain in preclinical studies, several phosphonate-coupled radiopharmaceuticals, such as samarium-153 complexes to tetraphosphonate, are expected to be effective pain palliation therapies for metastatic bone cancer and are currently being developed in clinical trials. Furthermore, recent reports on bisphosphonate-modified proteins have illustrated the feasibility of bone-specific delivery of biologically active protein drugs, such as cytokines and growth factors. ${ }^{17}$

\section{Hydrogels in Calcium Phosphate Moldableand Injectable Bone Substitute:}

As a consequence of trauma, disease and degeneration, the need for bone grafts is constantly growing. The limitations of auto-grafts consist mainly in explant site pain and morbidity, and limited availability. Allo and xeno-grafts can overcome these limitations, but the risk of immune reactions and, in some countries, limited availability of tissue banks, patient compliance and regulatory restrictions are major hurdles at present. In this respect, synthetic bone substitutes (SBSs) based on calcium phosphate $(\mathrm{CaP})$ materials are valid alternatives to tissue transplants, and their clinical use dates back more than a century. In the context of this paper, besides compounds synthesized via solution chemistry, thermal synthesis and thermal decomposition, CaPs also include semi-synthetic minerals such as deproteinized bovine bone. ${ }^{19}$

\section{Bone Grafts:}

There are four characteristics that an ideal bone graft material should exhibit which include:

$>$ Osteointegration, the ability to chemically bond to the surface of bone without an intervening layer of fibrous tissue;

$>$ Osteoconduction, the ability to support the growth of bone over its surface;

$>$ Osteoinduction, the ability to induce differentiation of pluripotential stem cells from surrounding tissue to an osteoblastic phenotype;

$>$ Osteogenesis, the formation of new bone by osteoblastic cells present within the graft material.

Only autogenous bone graft satisfies all of these requirements. Allograft is osteointegrative and osteoconductive and may exhibit osteoinductive potential, but it is not osteogenic because it contains no live cellular component. Synthetic bone graft substitutes currently possess only osteointegrative and osteoconductive properties. A variety of artificial materials has been used over the centuries to fill bone defects. Synthetic bone grafts at most possess only two of the four characteristics of an ideal bone graft material (osteointegration, osteoconduction).

$>$ Bioactive glasses: Two families of silicon-based compounds have the ability to bond directly to bone, bioactive glasses and the glass ionomers. Bioactive glasses are hard, solid (non-porous), materials that were first described in the 1970s. They consist of sodium oxide, calcium oxide, phosphorus pentoxide and silicon dioxide. Silicon dioxide (also known as silicate) forms the main component. Bioactive glasses possess both osteointegrative and osteoconductive properties.

$>$ Glass ionomers: Glass ionomer cements were first introduced in 1971 for dental use where cement was required to bind tooth enamel in a moist environment. The paste sets hard in approximately 5 min after which it is water insoluble. Prior to this it must be protected from wound fluids which will dissolve it.

$>$ Aluminium oxide: Alumina $\left(\mathrm{Al}_{2} \mathrm{O}_{3}\right)$ is a component of several bioactive materials but can serve as a bone graft substitute on its own. A mechanical bond occurs as a result of stresses on the implant that bring it into intimate relationship with the surrounding bone.

$>$ Calcium sulfate: Calcium sulfate is actually plaster of Paris. It was first documented as being used for fracture treatment by the Arabs in the 10th century, who would surround the affected limb in a tub of plaster. In 1852 a Dutch army surgeon by the name of Mathysen incorporated plaster into a bandageable forms (the form with which we are familiar today). Calcium sulfate is thought to act as an osteoconductive matrix for the ingrowth of blood vessels and associated fibrogenic and osteogenic cells.

\section{a. Antibiotic Beads:}

Antibiotic beads are both a complementary and alternative treatment option to intravenous antibiotic therapy for the treatment of osteomyelitis. 
Polymethylmethacrylate (PMMA) antibiotic beads were introduced clinically 30 years ago and have been the main local antibiotic delivery system in osteomyelitis therapy until recently. Polymethylmethacrylate antibiotic beads serve two functions in the treatment of osteomyelitis. Following surgical debridement of osteomyelitis, antibiotic-impregnated PMMA beads are surgically placed in the infected bone cavity to both sterilize and maintain dead space in the bone. Clearly, the use of antibiotic PMMA beads alone in osteomyelitis therapy offers several advantages. Studies reported these beads produce negligible to no systemic antibiotic serum concentrations, thereby leading to decreased systemic toxicity and adverse effects. Antibiotic-impregnated PMMA beads also decrease hospitalization duration and overall treatment cost. By eliminating the need for intravenous (IV) access, they also may increase both patient comfort and early ambulation. The use of PMMA as a delivery vehicle for antibiotic beads are nonbiodegradable.

\section{b. Biodegradable Local Drug Delivery Systems:}

New biodegradable drug delivery systems in development and testing offer hope in improving treatment for osteomyelitis. These new systems do not necessitate removal and should therefore eliminate the need for a second surgery. They will still serve to maintain the dead space in the bone and some may help facilitate new bone formation.

\section{a. Polylactic Acid:}

Polylactic acid (PLA) has been used for structural support following orthopedic procedures for years and has recently been studied as a delivery system for antibiotics. Previous use in humans for this purpose has produced rare adverse effects including non-infected draining sinuses and non-infected local inflammatory responses. The clinical significance of these adverse effects currently is unclear.

\section{b. Calcium Sulfate:}

Calcium sulfate is a promising vehicle that is biodegradable, obliterates dead space, and may aid in new bone formation. Animal model studies have assessed efficacy and safety, and early preliminary studies have been conducted in humans.

\section{Injectable Drug Delivery:}

The past decade has seen a dramatic shift in terms of the types of approved injectable drugs, the diseases they target, and the devices used to deliver them such as injectables bone cements for spinal coloum augmentation. The principal goal of applying these injectable bone cements is to augment impaired bony structures in order to maintain or improve the strength and stability of the spinal column. Currently, many new injectable bone cements are being designed specifically for application in the vertebral body. ${ }^{23}$

\section{Nanoparticles as a Tool For the Treatment of Skeletal System Disorders:}

In nanotechnology, a particle is defined as a small object that behaves as a whole unit with respect to its transport and properties. Particles are further classified according to diameter. Ultrafine particles or nanoparticles are sized between 1 and 100 nanometers. The reason for this double name of the same object is that, during the 197080 's, when the first thorough fundamental studies with "nanoparticles" were underway in the USA (by Granqvist and Buhrman) and Japan, (within an ERATO Project) they were called "ultrafine particles" (UFP).

Polymer nanoencapsulated rare earth aerogels- Rare earth (RE) aerogels combine the typical high porosity of aerogels with useful electrical, magnetic, optical and catalytic properties of the skeletal framework. RE aerogels are prepared by supercritical fluid $\mathrm{CO}_{2}$ drying of wet gels.

\section{CONCLUSIONS}

Skeletal drug delivery systems can be used to treat local and systemic diseases. The novel advancements in skeletal drug delivery with respect to materials, carriers and dosage forms open a wide era for development of better therapeutic dosage forms while minimising the drug, dose and the dosage. These will enhance the patient compliance for a much suitable therapeutic effect of the drug. 


\section{REFERENCES}

1. Jain AK and Panchagnula R, Skeletal drug delivery systems, International Journal of Pharmaceutics, 2000, 206(1-2), September, pp. 1-12.

2. Fauci A, Harrison's Rheumatology. Second Edition. McGrawHill Professional Publishing: Digital Edition, 2010, pp. 271.

3. Kumar V, Abbas AK, Fausto $\mathrm{N}$ and Mitchell RN, Robbins Basic Pathology. Eight edition. Saunders Elsevier, 2007, pp. 810-811.

4. Burnett MW, Bass JW and Cook BA, Etiology of osteomyelitis complicating sickle cell disease. Pediatrics, 1998, 101 (2), pp.296-297.

5. Ralston SH, Paget's Disease of Bone. New England Journal of Medicine, 368 (7) February, 2013, pp. 644-650.

6. Nelson JM, Chiller TM, Powers JH and Angulo FJ, Fluoroquinolone-resistant Campylobacter species and the withdrawal of fluoroquinolones from use in poultry: a public health success story. Clinical Infectious Disease, 2007, 44 (7), pp. 977-80.

7. Drlica K and Zhao X, DNA gyrase, topoisomerase IV, and the 4-quinolones. Microbiology and Molecular Biology Review, 1997, 61 (3), pp. 377-92.

8. Robinson MJ, Martin BA, Gootz TD, McGuirk PR and Osheroff N, Effects of novel fluoroquinolones on the catalytic activities of eukaryotic topoisomerase II: Influence of the C-8 fluorine group (PDF). Antimicrobial Agents and Chemotherapy, 1992, 36 (4), pp. 751-6.

9. Sissi $\mathrm{C}$ and Palumbo $\mathrm{M}$, The quinolone family: from antibacterial to anticancer agents. Current Medicinal and Chemical Anticancer Agents. 2003, 3 (6), pp. 439-50.

10. Hussy P, Maass G, Tümmler B, Grosse F and Schomburg U, Effect of 4-quinolones and novobiocin on calf thymus DNA polymerase alpha primase complex, topoisomerases I and II, and growth of mammalian lymphoblasts. Antimicrobial Agents Chemotherapy, 1986, 29 (6), pp. 1073-8.

11. Karande SC and Kshirsagar NA, Adverse drug reaction monitoring of ciprofloxacin in pediatric practice. Indian Pediatrician, 1992, 29 (2). pp. 181-8.
12. Pichichero ME, Use of selected cephalosporins in penicillinallergic patients: a paradigm shift. Diagnostic Microbiology and Infectious Disease, 2007, 57(3), pp. 13-18.

13. Anderson P, Samarium for osteoblastic bone metastases and osteosarcoma. Expert Opin Pharmacother, 2006, 7 (11), pp. 1475-86.

14. Ginebra MP, Traykova T and Planell JA, Calcium phosphate cements as bone drug delivery systems: a review. Journal of Controlled Release, 2006, 113(2), pp. 102-110.

15. Ginebra M, Traykova $\mathrm{T}$ and Planell JA, Mechanical and rheological improvement of a calcium phosphate cement by the addition of a polymeric drug. Journal of Biomedical Materials Research, 2001, 57 (1), pp. 113-118.

16. Yu D, Wong J, Matsuda Y, Fox JL, Higuchi WI and Otsuka M, Self-setting hydroxyapatite cement. Journal of Pharmaceutical Sciences, 1992, 81(6), June, pp. 529-531.

17. Hirabayashi $\mathrm{H}$ and Fujisaki J, (2003) Bone-specific drug delivery systems. Clinical Pharmacokinetics, 2003, 42(15), pp.1319-30.

18. Otsuka M, Nakahigashi Y, Matsuda Y, Fox JL and Higuchi WI, Effect of biological factors on indomethacin release from the cement loaded on bovine bone. Journal of Pharmaceutical Sciences, 2006, 83(11), November, pp. 1569-1573.

19. Charnley J, Arthroplasty of the hip - a new operation. Lancet, 1961, 277(7187), 1129-1132.

20. Boardman DR, Middleton FR and Kavanagh TG, A benign psoas mass following metal-on-metal resurfacing of the hip. Journal of Bone and Joint Surgery, 2006, 88(3), pp. 402-4.

21. Verlaan JJ, Lopez-Heredia MA and Jansen JA, Injectable Bone Cements for Spinal Column Augmentation: Materials for Kyphoplasty/Vertebroplasty, Comprehensive Biomaterials, 2011, Volume 6, pp. 147-160

22. Gauthier O, Bouler JM, Weiss P, Bosco J, Daculsi G and Aguado E, Kinetic study of bone ingrowth and ceramic resorption associated with the implantation of different injectable calcium-phosphate bone substitutes. Journal of Biomedical Material Research, 1999, 47, pp. 28-35. 